\title{
Determining the observer parameters for back EMF estimation for selected types of electrical motors
}

\author{
K. URBANSKI* \\ Institute of Control and Information Engineering, Poznan University of Technology, 3A Piotrowo St., 61-138 Poznan, Poland
}

\begin{abstract}
In the paper a procedure for determining the observer parameters for selected types of electrical motors is discussed. The procedure is based on the identity observer developed by Luenberger. The paper presents determining the parameters using calculation examples for the DC motor and also for the permanent magnet synchronous motor (PMSM), which utilize the extended state vector to estimate the back electromotive force (BEMF). Presented observer for PMSM does not need to use the information about load torque. The main task of this study is to show how to utilize these general theory to a specified type of motors. Such procedure avoids the use of time-consuming methods of the parameters selection, which are based on random algorithms or the computational intelligence.
\end{abstract}

Key words: permanent magnet motors, DC motors, sensorless control, observers.

\section{Introduction}

Many electrical drives operate in regime of position control, speed control or current control. To take full advantage of position control or speed control, the shaft position sensor or/and speed sensor has to be used, especially in a vector control of AC drives. However, the usage of those sensors in general increases weight, size and cost of the drive. The control of electrical motors takes advantage of sensorless control in wherever the compact design, high reliability, and damage resistance are the important features of the drive. The sensorless operation mode may be a goal in itself or may be the emergency solution in a case of sensor malfunction. One of the many methods to determine or control the speed and the position is estimation of the unknown state variable or the use of the dual observer $[1,2]$. For this purpose a various method may be used, e.g. methods based on the Luenberger observer [1] and modified Luenberger observer [3], sliding mode observer [4], or methods which use Kalman filter [5, 6]. Expansion of the correction function of Luenberger observer from proportional form into form of proportional-integral improves observer's performance in case of rapid changes of speed and improves robustness on electromagnetic disturbances [7]. However, such structure of the observer is little more difficult for theoretical analysis. In order to determine the observer parameters, various methods are presented in the literature: most commonly are experimental methods of finding the parameters or without information about method [8-10], using different searching algorithms, e.g. random or genetic algorithm [11] which are based on quality function or methods based on Lyapunov function $[12,13]$. Typically, position estimation methods are based on the BEMF estimation at the first stage, and then, in a second stage, using obtained

*e-mail: konrad.urbanski@put.poznan.pl

Manuscript submitted 2016-10-12, revised 2017-01-12, initially accepted for publication 2017-01-17, published in August 2017.
BEMF values the position is calculated. This paper focuses on this first stage and especially on the determining the observer parameters. An easy form of the procedure is presented including calculation examples.

\section{The algorithm}

To estimate the unknown variables of the state vector for system (1):

$$
\begin{gathered}
\dot{\mathbf{x}}=\mathbf{A} \cdot \mathbf{x}+\mathbf{B} \cdot \mathbf{u} \\
\mathbf{y}=\mathbf{C} \cdot \mathbf{x}
\end{gathered}
$$

it may be used the Luenberger observer:

$$
\hat{\dot{\mathbf{x}}}=\mathbf{A} \cdot \hat{\mathbf{x}}+\mathbf{B} \cdot \mathbf{u}+\mathbf{G} \cdot(\mathbf{y}-\mathbf{C} \cdot \hat{\mathbf{x}})
$$

where symbol $\wedge$ means estimated variables. To get proper operation (commonly understood as fast and stable) of the sensorless drive, it is important to determine the suitable set of the observer parameters - the observer input vector $\mathbf{G}$. Based on the procedure presented in [1], the process of determining the observer parameters can be summarized up in a few simple steps. First, to perform the observer parameters calculations using these procedure, the system should be represented as a state space model. It requires to determine system matrix $\mathbf{A}$, and output matrix C. Then, the observer input vector $\mathbf{G}$ is determined. Next, the observer system matrix (OSM) is calculated using (3):

$$
\mathbf{O S M}=\mathbf{A}-\mathbf{G} \cdot \mathbf{C}
$$

and characteristic polynomial of OSM is calculated using wellknown dependence:

$$
\operatorname{POSM}(s)=\operatorname{det}|s \cdot \mathbf{I}-\mathbf{O S M}|
$$


where I denotes the identity matrix, the same size as OSM. Now, proposed dynamics of estimation error decay is declared by reference polynomial $P_{r e f}(s)$. If $(\mathbf{C}, \mathbf{A})$ is completely observable, we can find the observer's gain matrix $\mathbf{G}$, which gives possibility to get arbitrary determined the dynamics of the observer by solving the relationship (5):

$$
\operatorname{POSM}(s)=P_{\text {ref }}(s)
$$

\section{Calculation examples}

Some examples of calculating the parameters of the BEMF observer will follow. Simulations were performed using Matlab-Simulink environment and calculations using Mathcad Prime environment. The first example shows detailed procedure to determine observer parameters for DC motor. The state vector contains, as usually, the armature current and the shaft speed. It is direct derivation from the Luenberger's method; however, it is important to understand the method and also for understanding next step - reduction of the analyzed model to the only electrical part of the motor model. The state vector now contains the armature current and the BEMF. An observer estimates the BEMF (which gives directly the mechanical value - the speed, using output matrix C) without using the mechanical part of the motor model. It is advantageous because of no need to measure or estimate the motor load. Such model may be finally converted to suitable structure corresponding to the permanent magnet synchronous motor. It is the most important example. As in the previous case, there is a method which utilizes the extension of the state vector by the BEMF. For such modified object, the Luenberger observer may be used, basing on the analytical way of the calculating the observer's gain matrix.

3.1. DC motor - full order observer. Let us consider the linear, time-invariant motor model, presented in Fig. 1. The part of the scheme selected by dashed line will be discussed in the next paragraph. Such structure - named here $D C 1$ - corresponds to the system which may be presented as the state space model A, B, C (6), with state vector and input vector defined as (7):

$$
\begin{gathered}
\mathbf{A}=\left[\begin{array}{rr}
-\frac{R}{L} & -\frac{k \Phi}{L} \\
\frac{k \Phi}{J} & 0
\end{array}\right], \quad \mathbf{B}=\left[\begin{array}{rr}
\frac{1}{L} & 0 \\
0 & -\frac{1}{J}
\end{array}\right], \mathbf{C}=\left[\begin{array}{ll}
1 & 0
\end{array}\right] \\
\mathbf{x}=\left[\begin{array}{c}
i \\
\omega
\end{array}\right], \mathbf{u}=\left[\begin{array}{c}
u_{S} \\
T_{L}
\end{array}\right] .
\end{gathered}
$$

The variables $R, L, k \Phi, J$ means armature resistance and inductance, flux constant and total moment of inertia - respectively. The $\mathbf{x}$ is the state vector which contains $i$ - armature current, $\omega$ - the shaft speed, $\mathbf{u}$ is the input vector where $u_{s}$ is the supply voltage and $T_{L}$ is load torque. To prepare properly the observer, output vector of motor model contains only current, because in assumption the speed is not available to the measurement. This results in present form of matrix $\mathbf{C}$. Now, the input vector $\mathbf{G}$ of the observer is specified as:

$$
\mathbf{G}_{D C 1}=\left[\begin{array}{c}
g_{i} \\
g_{\omega}
\end{array}\right]
$$

where $g_{i}$ and $g_{\omega}$ means observer gains for correcting loop of current, and of speed (instead - in this example - of the BEMF), accordingly. For parameter set:

$$
\begin{array}{ccrl}
R=1.25 \Omega & L & =10 \mathrm{mH} \\
J=0.11 \mathrm{~kg} \cdot \mathrm{m}^{2} & k \Phi & =2.23 \mathrm{~Wb}
\end{array}
$$

according to $(3,6,8)$, the resulting $\mathbf{O S M}$ has form:

$$
\mathbf{O S M}_{D C 1}=\left[\begin{array}{cc}
-g_{i}-125 & -223 \\
-g_{\omega}+20.27 & 0
\end{array}\right]
$$

and its characteristic polynomial has the following form:

$P_{\mathrm{OSM} D C 1}(s)=s^{2}+\left(g_{i}+125\right) \cdot s+4520.2-223 \cdot g_{\omega} \cdot(11)$

Now, designed dynamics of the estimation error decay is chosen. Let's assume that the dynamics may be described by (12):

$$
P_{r e f D C 1}(s)=s^{2}+400 \cdot s+40000
$$

Solving equation (5) substituting (11) and (12), yields:

$$
g_{i}=275 \quad g_{\omega}=-159.1 .
$$

Finally, the testing scheme is proposed in Fig. 2. The model of DC motor (with structure as in Fig. 1) is shown; and the observer, presented as the Simulink model. Calculated values of matrices $\mathbf{A}, \mathbf{B}$ and $\mathbf{G}$, are shown in this figure. Simple testing procedure is used: direct start of the motor and at time $0.15 \mathrm{~s}$ the load is attached. Only in order to verify operation of the observer, an additional control path is used. It consist of block "disruption", which generates step change of the disrupting signal for estimated values. The other block named "tester"

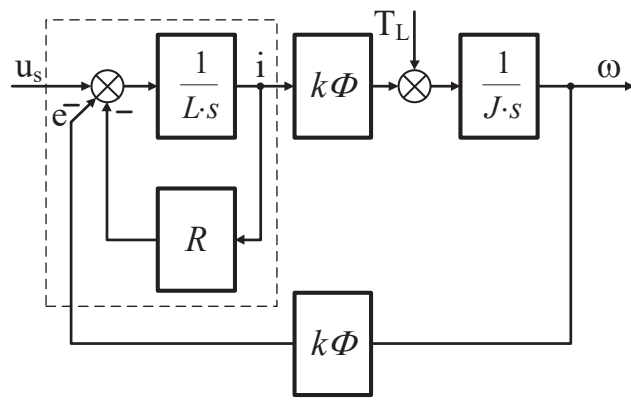

Fig. 1. General view of the DC motor model 


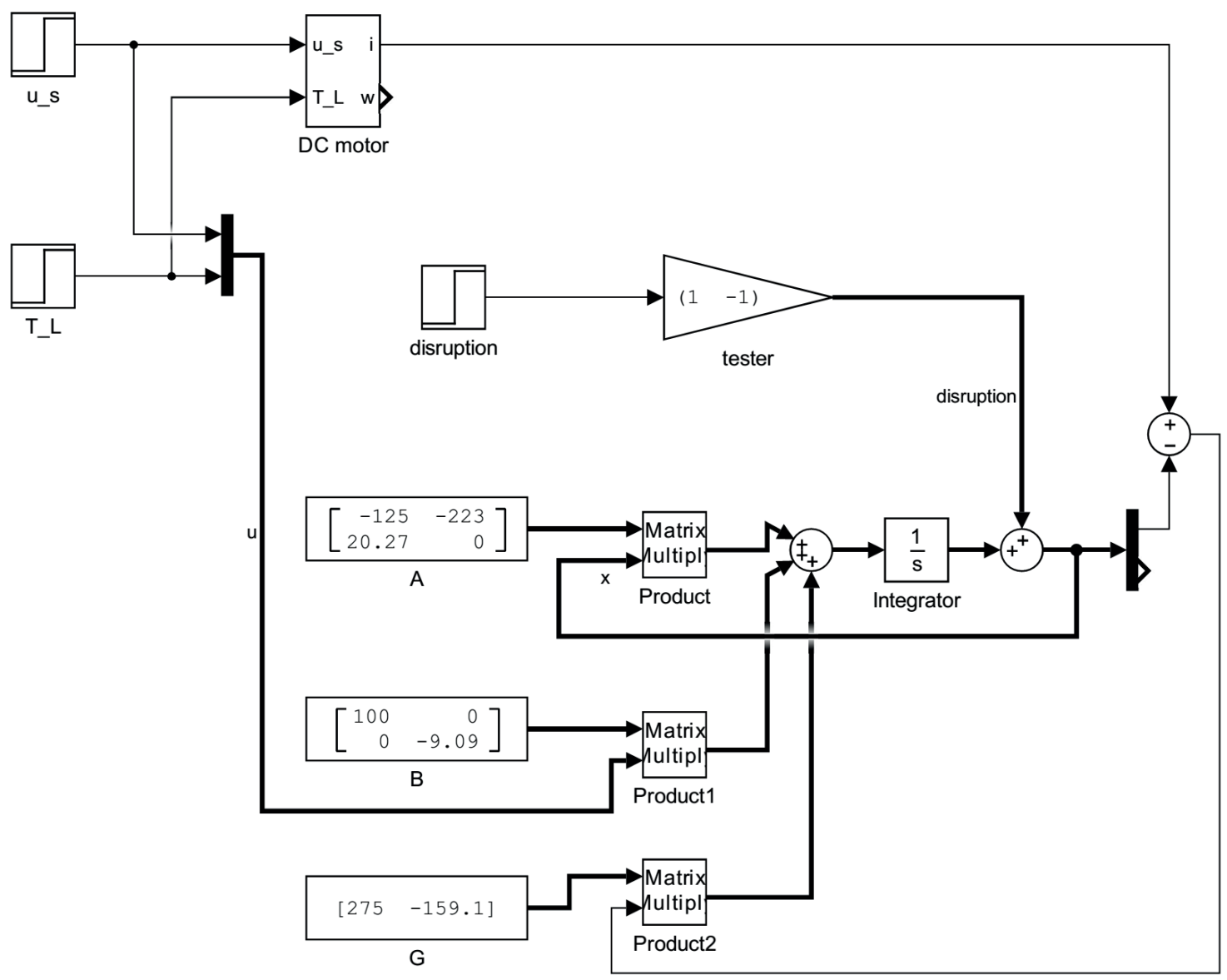

Fig. 2. Verifying model for the observer parameters calculations for DC motor prepared in Matlab-Simulink

is a switch which controls the presence and the sign of the disruption, respectively for current correcting loop and for speed correcting loop. Value $\left[\begin{array}{ll}0 & 0\end{array}\right]$ means "no disruption". In the example, the resultant waveforms for direct connection to supply are shown in Fig. 3, where a constant value of disruption (step $0 \rightarrow 10 \mathrm{~A}$ and $0 \rightarrow-10 \frac{\mathrm{rad}}{\mathrm{s}}$ ) is attached at the time $0.3 \mathrm{~s}$.
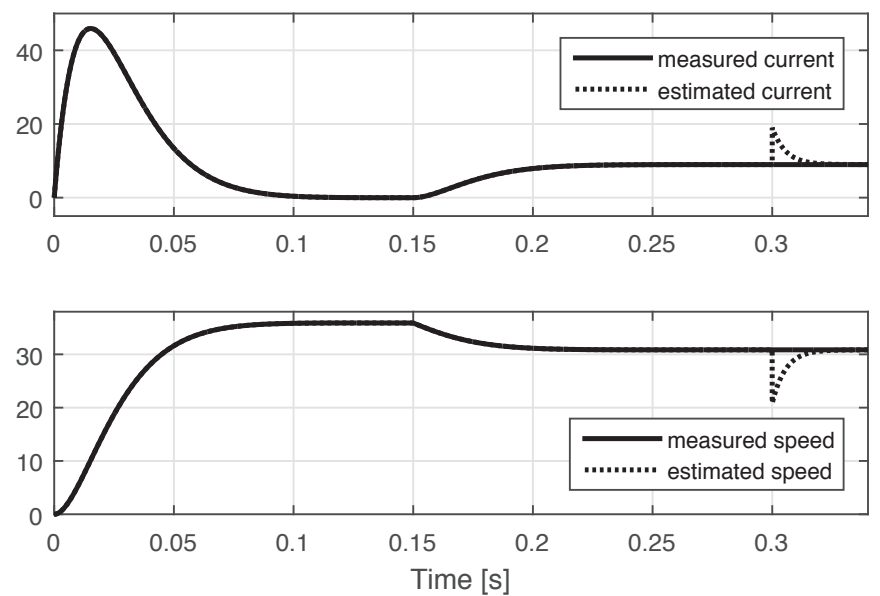

Fig. 3. The waveforms of measured and estimated current and speed using presented observer parameters - test $D C 1$

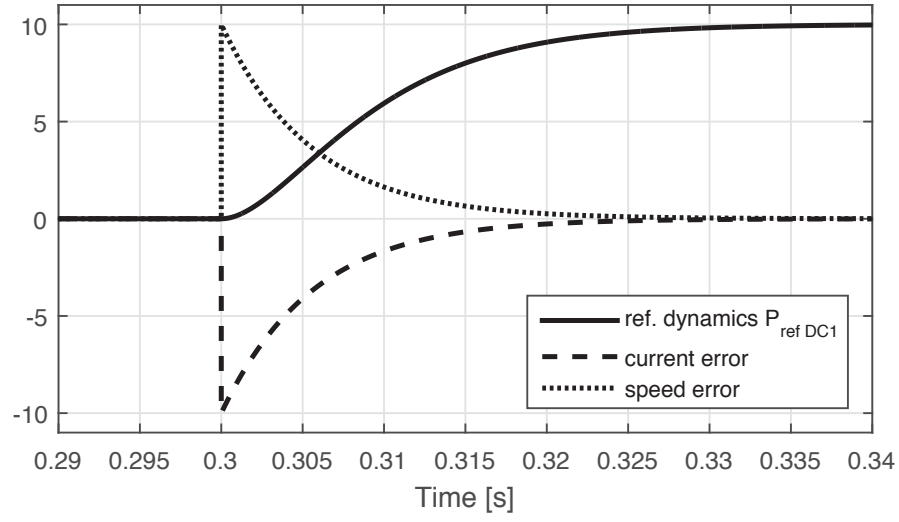

Fig. 4. The step response waveforms, on estimation disruption for designed the observer - test $D C 1$

In general, in such case, estimated and measured waveforms overlap. The enlarged part of the observer performance with the disruption introduction is shown in Fig. 4. The referenced dynamics defined by $P_{r e f D C 1}$ is visible as a step response printed as a solid line (waveform \#1). The dashed and doted lines are the estimation error waveforms for current and speed. It is visible that designed dynamics of the observer is fulfilled and all three waveforms decay at the same (designed) time. 
3.2. DC motor - reduced order. As it was mentioned in the previous paragraph, in order to use of the full order observer, the information about torque load is required for proper operation. It is possible to eliminate the mechanical part of the motor in the observer structure, which reduces number of the inputs of that observer to the electrical variables only. Neither the load torque nor the moment of inertia does not have to be identified. If such separated electrical part of the motor (dashed line in Fig. 1) is to be used in the observer structure, they must be properly interpreted. It is proposed that in place of calculated BEMF from the speed through $k \Phi$ gain, the BEMF is calculated

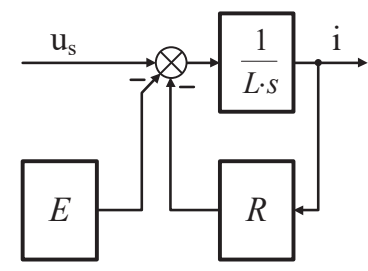

Fig. 5. General view of the electrical part of DC motor model used to design the observer only using the correction loop of the observer. It means, that at considered calculation step, the BEMF derivative is equal zero - the BEMF at considered calculation step is the constant. So, the model, which is used to design the observer, has the form presented in Fig. 5. The $E$ value means constant at considered calculation step the BEMF value. Such structure corresponds to the system which may be presented as the state space model described by the matrices A, B, C (14):

$$
\mathbf{A}=\left[\begin{array}{rr}
-\frac{R}{L} & -\frac{1}{L} \\
0 & 0
\end{array}\right], \quad \mathbf{B}=\left[\begin{array}{l}
\frac{1}{L} \\
0
\end{array}\right], \quad \mathbf{C}=\left[\begin{array}{ll}
1 & 0
\end{array}\right]
$$

Now, the state vector and input vector have the following form:

$$
\mathbf{x}=\left[\begin{array}{l}
i \\
e
\end{array}\right], \quad \mathbf{u}=\left[\begin{array}{l}
u_{s}
\end{array}\right]
$$

where $e$ means back electromotive force. It is noticeable that second row in matrices $\mathbf{A}$ and $\mathbf{B}$, which is responsible for changing the $e$ value, is set to zero, and according to (2) only

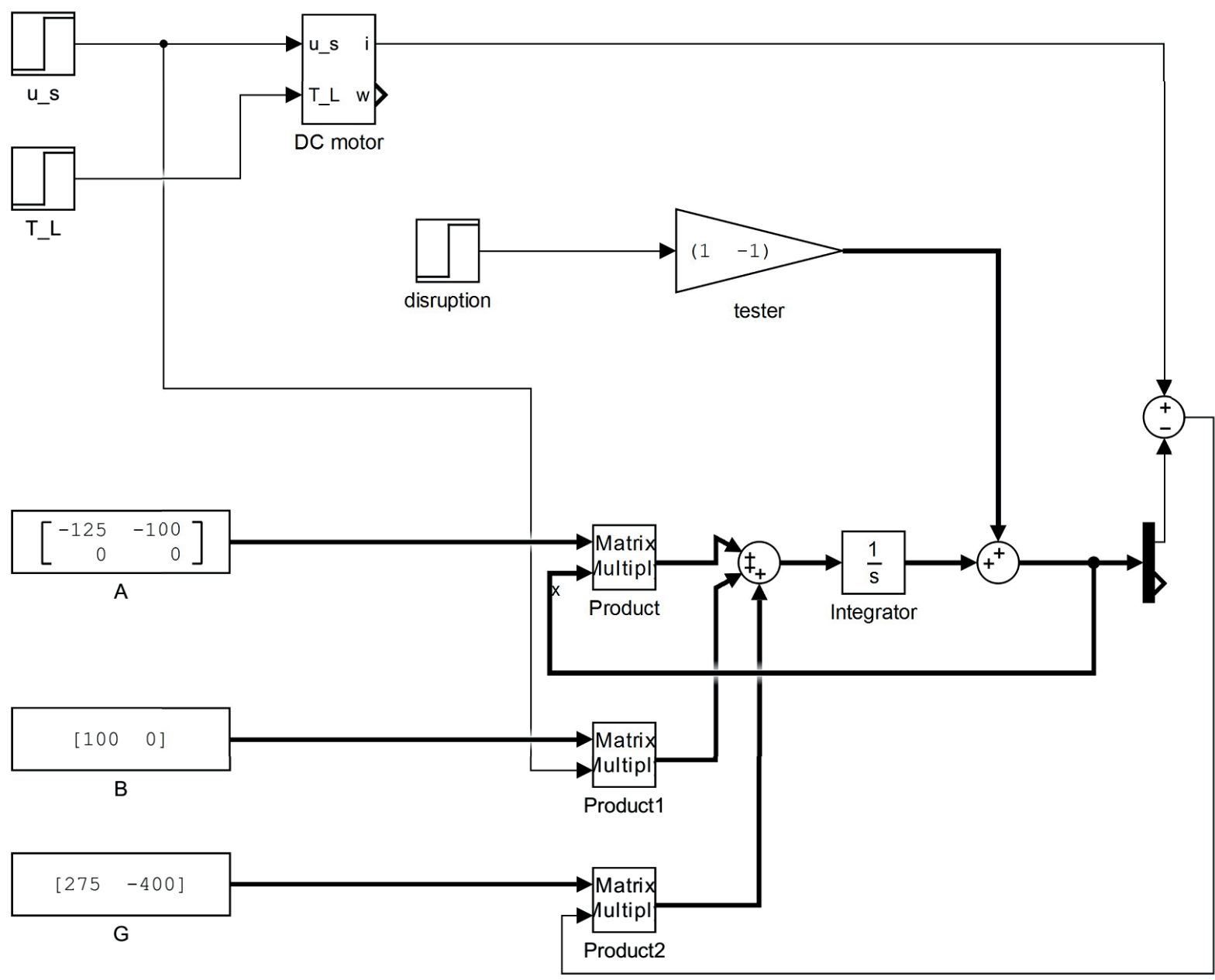

Fig. 6. Verifying model for the observer parameters calculations for DC motor prepared in Matlab-Simulink in a case of reduced model 

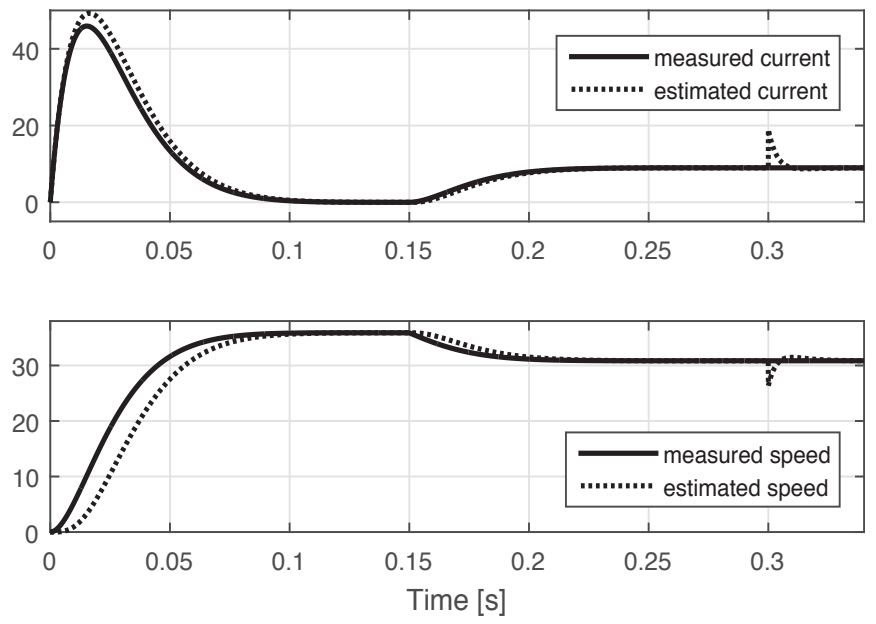

Fig. 7. The waveforms of measured and estimated current and speed using presented observer parameters - test $D C 2$

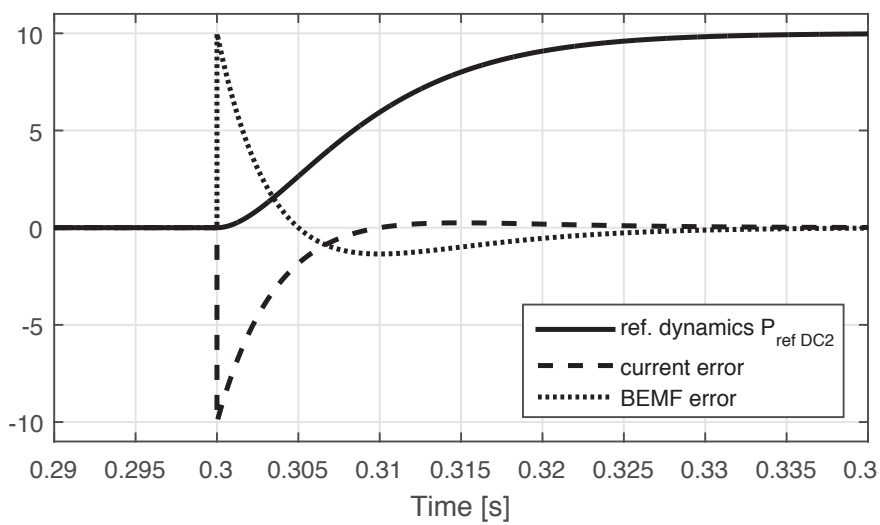

Fig. 8. The step response waveforms, on estimation disruption for designed the observer - test $D C 2$

non-zero gain of input vector $\mathbf{G}$ may produce the estimated value of $\hat{e}$. The system has been named $D C 2$. Suppose that the $\mathbf{G}$ is specified as:

$$
\mathbf{G}_{D C 2}=\left[\begin{array}{l}
g_{i} \\
g_{e}
\end{array}\right]
$$

where $g_{i}$ and $g_{e}$ means observer gains for correcting loop of current, and of BEMF, accordingly. Using the same parameters set as in previous example (9), the $\mathbf{O S M}$ is determined according to $(3,14,16)$ :

$$
\mathbf{O S M}_{D C 2}=\left[\begin{array}{cc}
-g_{i}-125 & -100 \\
-g_{e} & 0
\end{array}\right]
$$

and its characteristic polynomial has the following form:

$$
P_{\text {OSM } D C 2}(s)=s^{2}+\left(g_{i}+125\right) \cdot s-100 \cdot g_{e} .
$$

Let's assume that the dynamics may be the same as previous, $P_{\text {ref } D C 2}=P_{\text {refDC1 }}$ (12). Solving equation (5) substituting (18) and (12), yields:

$$
g_{i}=275 \quad g_{e}=-400 .
$$

Similar to previous example the testing scheme (Fig. 6) and testing procedure is used in this case. The DC motor parameters are the same as previous, the differences are smaller size of input vector $\mathbf{u}$, other parameters of the observer's model, and the observer's input matrix G. The resulting waveforms are presented in Figs. 7 and 8. Now, the situation is different. The measured and estimated waveforms of current and speed (speed is calculating by divide the $\hat{e}$ by $k \Phi$ ) are not overlapped at transients, even in a case of zero initial error of current and speed. It is noticeable that the decay of the estimation error has different character than previously; however, the decay time of errors is equal to designed value (Fig. 8).

3.3. Observer for PMSM using extended state vector. To simplify the model, it is assumed that there is no windings in rotor, eddy currents and the effect of temperature is neglected, the flux produced by the rotor is constant [14]. It is assumed also the magnetic symmetry of the motor. In a case of motor model form, which is prepared to use in design the observer, it is convenient to use such form, which may be used without converting the coordinates systems using just estimated position (which is calculated from BEMF). That's why the motor model is written in stationary $\alpha \beta$ coordinates system. It ensures reliable conversion from three phase $a b c$ system to observer's coordinates system, by means of Clarke transformation [15]. So, the PMSM model chosen to use in the observer has the following form (20-23):

$$
\begin{gathered}
\frac{d i_{\alpha}}{d t}=-\frac{R_{s}}{L_{s}} \cdot i_{\alpha}-\frac{1}{L_{s}} \cdot e_{\alpha}+\frac{1}{L_{s}} \cdot u_{\alpha} \\
\frac{d i_{\beta}}{d t}=-\frac{R_{s}}{L_{s}} \cdot i_{\beta}-\frac{1}{L_{s}} \cdot e_{\beta}+\frac{1}{L_{s}} \cdot u_{\beta} \\
e_{\alpha}=-k_{e} \cdot \Psi_{f} \cdot \omega \cdot \sin (\Theta) \\
e_{\beta}=k_{e} \cdot \Psi_{f} \cdot \omega \cdot \cos (\Theta) \\
\Psi_{\alpha}=L_{s} \cdot i_{\alpha}+\Psi_{f} \cdot \cos (\Theta) \\
\Psi_{\beta}=L_{s} \cdot i_{\beta}-\Psi_{f} \cdot \sin (\Theta) \\
\frac{d \omega}{d t}=\frac{1}{J} \cdot\left(\Psi_{\beta} \cdot i_{\alpha}-\Psi_{\alpha} \cdot i_{\beta}-T_{L}\right) \\
\frac{d \Theta}{d t}=\omega
\end{gathered}
$$

where $u_{\alpha}, u_{\beta}$, means the components of input voltage, $i_{\alpha}, i_{\beta}$, the components of currents, $\Psi_{\alpha}, \Psi_{\beta}, e_{\alpha}$ and $e_{\beta}$, means flux and BEMF in the $\alpha \beta$ axis respectively, and $\Psi_{f}$ means flux excited by permanent magnet. The load torque is represented by symbol 


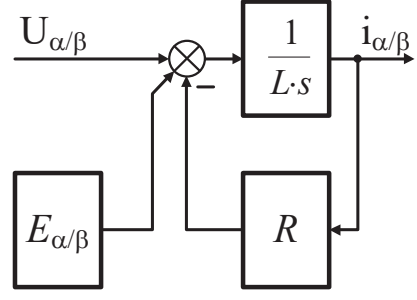

Fig. 9. Part of PMSM model used to prepare BEMF observer

$T_{L}, \omega$ is the mechanical speed. The $R_{S}$ means the windings resistance, $L_{s}$ means the windings inductance, $\Theta$ means shaft position, and $J$ is the total moment of inertia. The motor model with state variables $i_{\alpha}, i_{\beta}, \omega$ and $\Theta$ is non-linear. However, all state variables are measurable, to avoid the use of mechanical sensor, the speed and position should be estimated. For instance, it is possible to estimate the BEMF and then calculate the shaft position and/or speed. According to the method presented in [16] it is convenient to use only first two electrical equations (20), in which the BEMF components are considered as disturbances. Hence there is no need to identify $J, \Psi_{f}$ and also the $T_{L}$. The part of the PMSM model, which is used to prepare the BEMF observer, has the form presented in Fig. 9. It can be noticed that this structure is similar to structure presented in Fig. 5. The state vector and input vector have been presented below accordingly:

$$
\mathbf{x}_{\alpha / \beta}=\left[\begin{array}{c}
i_{\alpha / \beta} \\
e_{\alpha / \beta}
\end{array}\right], \quad \mathbf{u}_{\alpha / \beta}=\left[u_{\alpha / \beta}\right]
$$

For that system the Luenberger observer is used. In presented realization, the proportional type of correction is used. It utilizes the error between measured and estimated value of the currents. In a case of the assumption of the magnetic symmetry, the observer may have the same form for both $-\alpha$ and $\beta$ coordinate axes, and is easily realized as two independent observers for every axis. Such form, without joining the axis matrices together into one bigger matrix, may be easier in some applications in a case of programing for microprocessor systems without matrix maths. Assuming that at considered calculation step, the BEMF derivative is equal zero (as was made in section 3.2), one can write the observer equations as (25-28):

$$
\begin{aligned}
& \frac{d \hat{i}_{\alpha}}{d t}=-\frac{R_{s}}{L_{s}} \cdot \hat{i}_{\alpha}-\frac{1}{L_{s}} \cdot \hat{e}_{\alpha}+\frac{1}{L_{s}} \cdot u_{\alpha}+g_{i}\left(i_{\alpha}-\hat{i}_{\alpha}\right) \\
& \frac{d \hat{i}_{\beta}}{d t}=-\frac{R_{s}}{L_{s}} \cdot \hat{i}_{\beta}-\frac{1}{L_{s}} \cdot \hat{e}_{\beta}+\frac{1}{L_{s}} \cdot u_{\beta}+g_{i}\left(i_{\beta}-\hat{i}_{\beta}\right)
\end{aligned}
$$

$$
\begin{aligned}
& \frac{d \hat{e}_{\alpha}}{d t}=g_{e}\left(i_{\alpha}-\hat{i}_{\alpha}\right) \\
& \frac{d \hat{e}_{\beta}}{d t}=g_{e}\left(i_{\beta}-\hat{i}_{\beta}\right)
\end{aligned}
$$

where $g_{i}$ is the estimated currents gain, and $g_{e}$ is the estimated BEMF gain. This set of equations (25-28) may be presented in the form of matrix equation:

$$
\hat{\dot{\mathbf{x}}}=\mathbf{A} \cdot \hat{\mathbf{x}}+\mathbf{B} \cdot \mathbf{u}+\mathbf{G}[\Delta \mathbf{i}]
$$

where $\mathbf{G}$ is an array of the correction gains (observer's input vector), and $\Delta i=i-\hat{i}$. Considering this configuration as a modification of system from section 3.2, for every axis of the coordinates system, it is possible to create the independent state space model to use as the observer in form (14). The observer parameters depend only on the PMSM parameters, and observer's performance is independent of the control methods of the PMSM. However, it should be mentioned that the sensorless drive working quality may depend on type of controllers or their settings, due to estimation error dependency to the dynamics of the BEMF. As used in an example, the control structure is typical for vector control method, with inner current control loop in $d q$ coordinates system and with outer speed control loop. The waveforms show the observer the performance in a case of parameter selection using proposed method. The part of the control structure used for BEMF estimation is shown in Fig. 10, where obs_alpha and

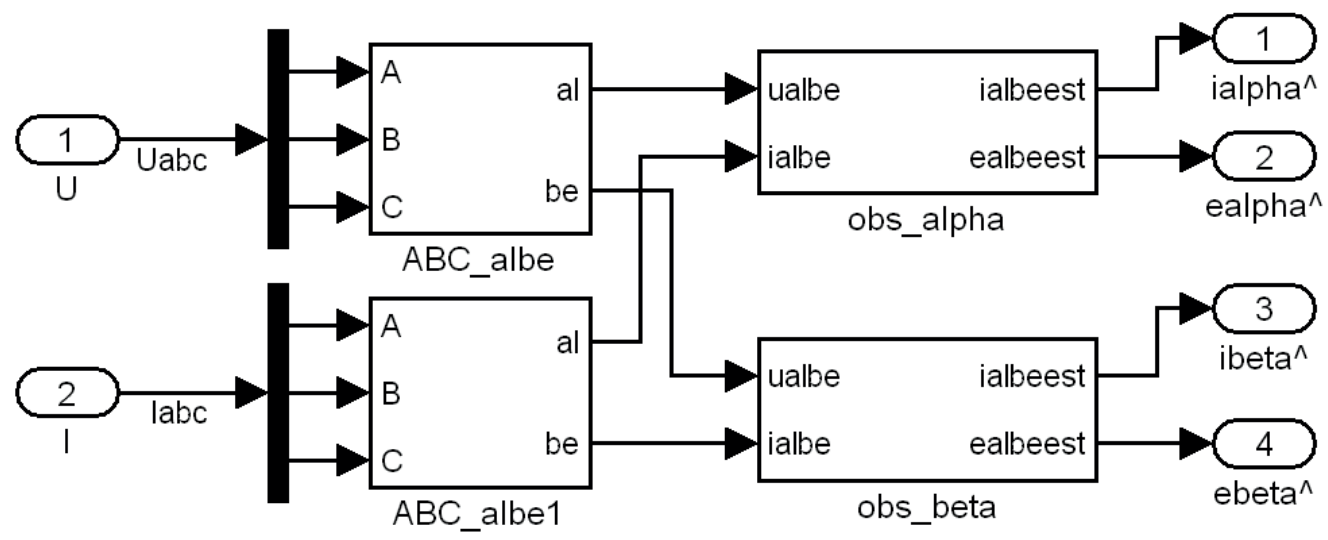

Fig. 10. Part of the control structure of PMSM with estimation structure of BEMF, which consist of two independent observeres for $\alpha$ and $\beta$ axis 


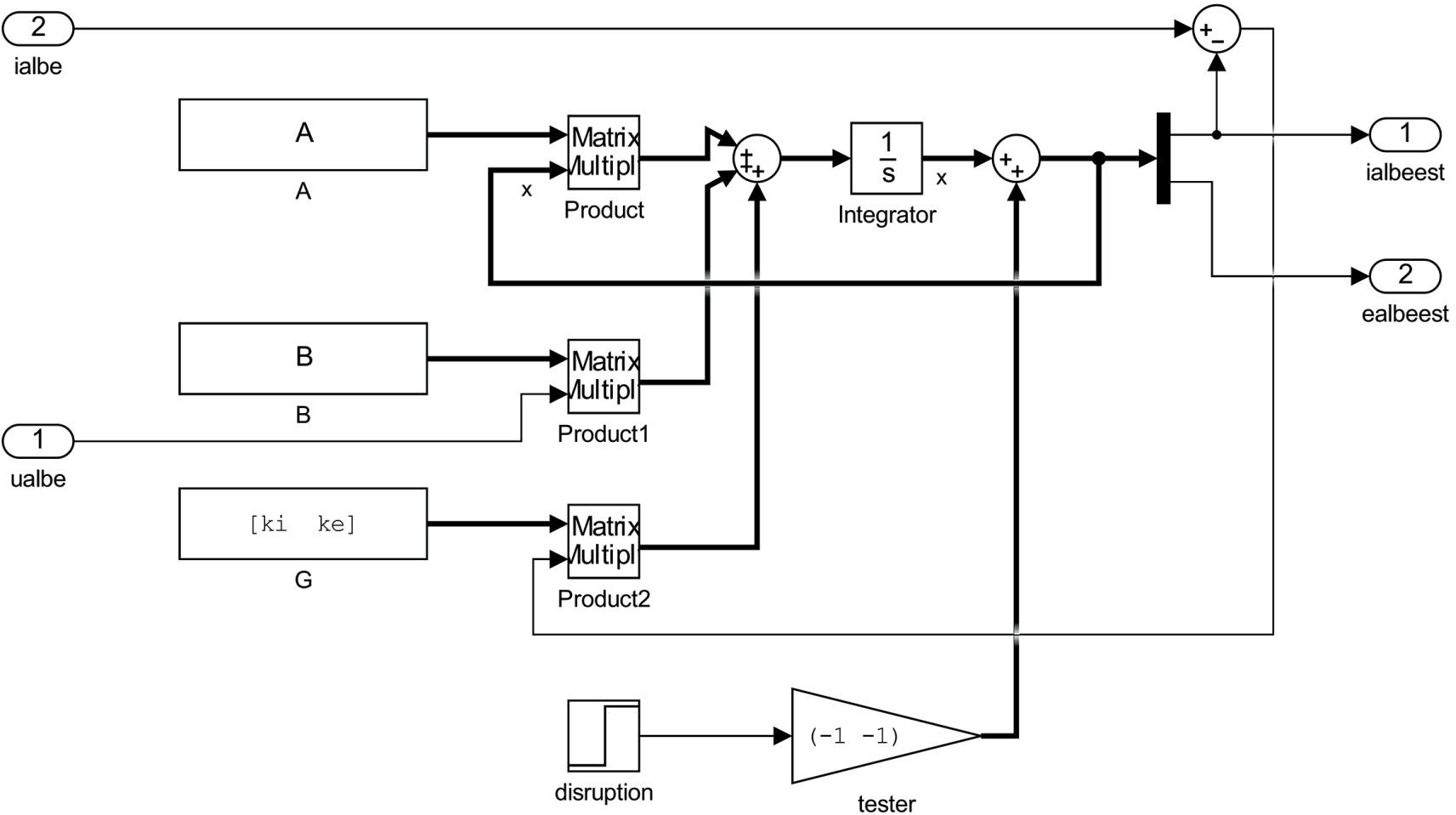

Fig. 11. Verifying model for the observer parameters calculations for PMSM prepared in Matlab-Simulink for one axis

obs_beta have inner structure presented in Fig. 11. Signals at inputs 1 and 2 (Fig. 10) are measured voltages and currents, signals at outputs $1-4$ are the estimated (marked as ${ }^{\wedge}$ ) values of current and BEMF in axes $\alpha$ and $\beta$. Similar to Section 3.2, the observer has the same form (Fig. 11) in both axis $\alpha$ and $\beta$. To verify the decay of the estimation error, similar to previous example the testing scheme, and similar testing procedure is used.

Consider the PMSM drive, where maximal speed is equal $100 \frac{\mathrm{rad}}{\mathrm{s}}$. It follows that in assumption the one pair of poles, the fastest BEMF period (the worst case from the point of view of the observer operation) covers about $63 \mathrm{~ms}$. The proper design of the observer's dynamics is a very important stage in case of use the observer as a part of sensorless control in the closed mode, where estimated values are used in the control chain. The open mode in the context of sensorless operation means that the estimated values are used only to view the not measured state variables. Presented below waveforms were prepared in open mode, in order to preserve clean operation of an observer. The control system which is used, it is the typical field oriented control (FOC) method for the PMSM. It covers cascade control structure, using closed speed control loop with inner current control loops. They contain separate controllers in $d$ and $q$ axis, and also a PWM inverter with carrier frequency equal $10 \mathrm{kHz}$. Drive model was prepared using SimPowerSystems library of Matlab-Simulink.

Suppose also that about $1 / 20$ of BEMF period ( $3 \mathrm{~ms}$ ) gives enough time to get acceptable accuracy of BEMF estimation at steady state of the drive. For the considered system, the simplified method to find the characteristic polynomial, involves finding the parameter $a$ of equation $(s-a)^{2}$ which gives the settling time as close as possible to designed value
( $3 \mathrm{~ms}$ ). In a case of $a=-3200$, the designed dynamics may be described as:

$$
\operatorname{PrefPMSM}_{\operatorname{PM}}(s)=s^{2}+6400 \cdot s+10240000
$$

and its step response is presented in Fig. 14 ("norm ref. dynamics" - waveform \#1) where step change of estimation error is involved at time $t=0.1 \mathrm{~s}$ (Fig. 13-15).

For considered parameter set:

$$
R_{s}=0.7 \Omega \quad L_{s}=5.7 \mathrm{mH}
$$

the calculated matrices have form:

$$
\begin{gathered}
\mathbf{A}_{\alpha / \beta}=\left[\begin{array}{cc}
-122.1 & -174.4 \\
0 & 0
\end{array}\right], \\
\mathbf{B}_{\alpha / \beta}=\left[\begin{array}{c}
174.4 \\
0
\end{array}\right], \quad \mathbf{C}_{\alpha / \beta}=\left[\begin{array}{ll}
1 & 0
\end{array}\right] .
\end{gathered}
$$

Now, because of assumption of magnetic symmetry in $\alpha$ and $\beta$ axes, the $\mathbf{G}$ matrix is specified similar as (16):

$$
\mathbf{G}_{P M S M}=\left[\begin{array}{l}
g_{i} \\
g_{e}
\end{array}\right]
$$

and according to $(3,32,33)$, the resulting OSM has form:

$$
\mathbf{O S M}_{P M S M}=\left[\begin{array}{cc}
-g_{i}-122.1 & -174.4 \\
-g_{e} & 0
\end{array}\right]
$$


Characteristic polynomial of $\mathbf{O S M}_{P M S M}$ is presented below:

$$
P_{\text {OSMPMSM }}(s)=s^{2}+\left(g_{i}+122.1\right) \cdot s-174.4 \cdot g_{e} .
$$

Solving equation (5) substituting (35) and (30), yelds:

$$
g_{i}=6278 \quad g_{e}=-58709 .
$$

In this case, testing procedure includes operation of PMSM using FOC and the set of reference speed step changes, as is shown in Fig. 12. At time equal $0.1 \mathrm{~s}$ a step disturbance in current correcting loop and also in BEMF correcting loop was introduced as shown in Fig. 11. In Fig. 13 waveforms of BEMF and their estimated values are shown, according to the speed trajectory from Fig. 12. It is visible that the proper estimation of BEMF and its accuracy strongly depends on speed and the designed dynamics of the observer. This is because observed values still follow the constantly changing BEMF values. It is

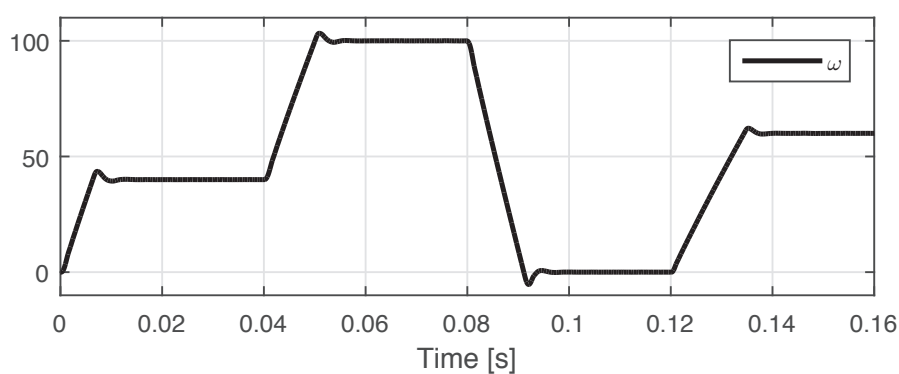

Fig. 12. Measured speed $\omega$ waveform obtained for set of step change of reference speed $\omega_{\text {ref }}: 0 \rightarrow 40 \rightarrow 100 \rightarrow 0 \rightarrow 60 \frac{\mathrm{rad}}{\mathrm{s}}$. This figure is the reference for Fig. 13. Test PMSM
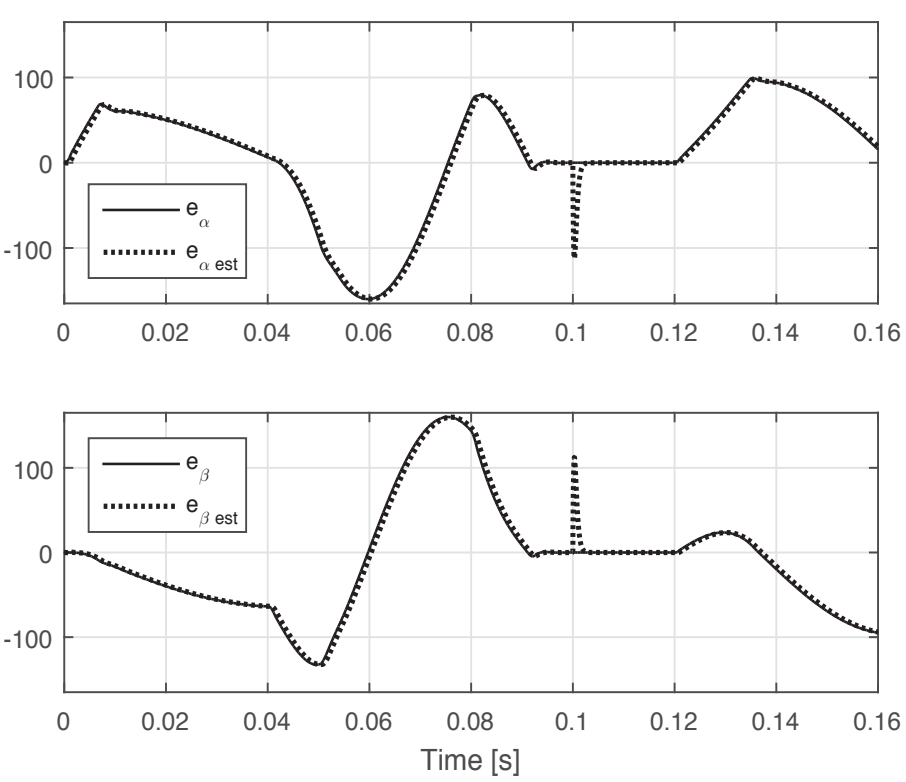

Fig. 13. Waveforms of BEMF and theirs estimated values according to Fig. 12: up - BEMF in $\alpha$ axis and its estimated value, down - BEMF in $\beta$ axis and its estimated value - test PMSM

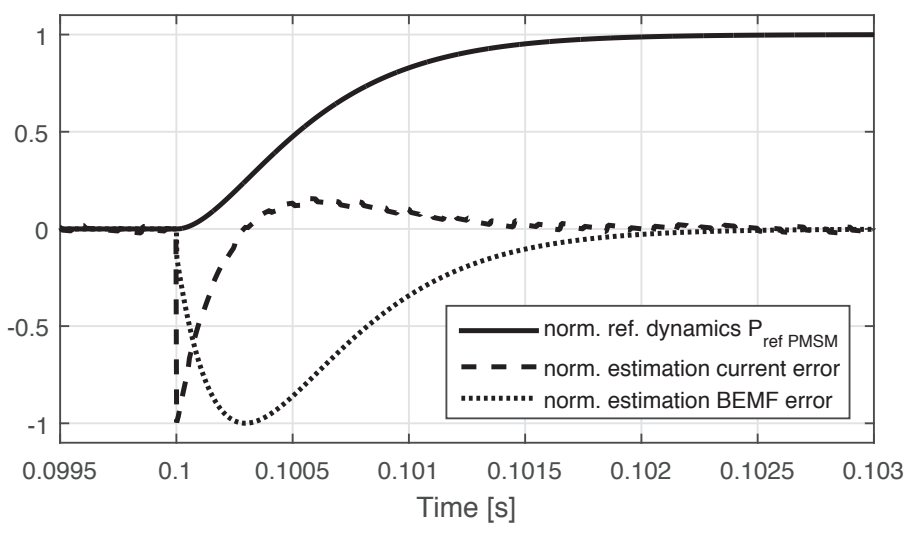

Fig. 14. Zoomed and normalized view for step response waveforms on estimation disruption, for one phase $(\alpha)$ - simulation

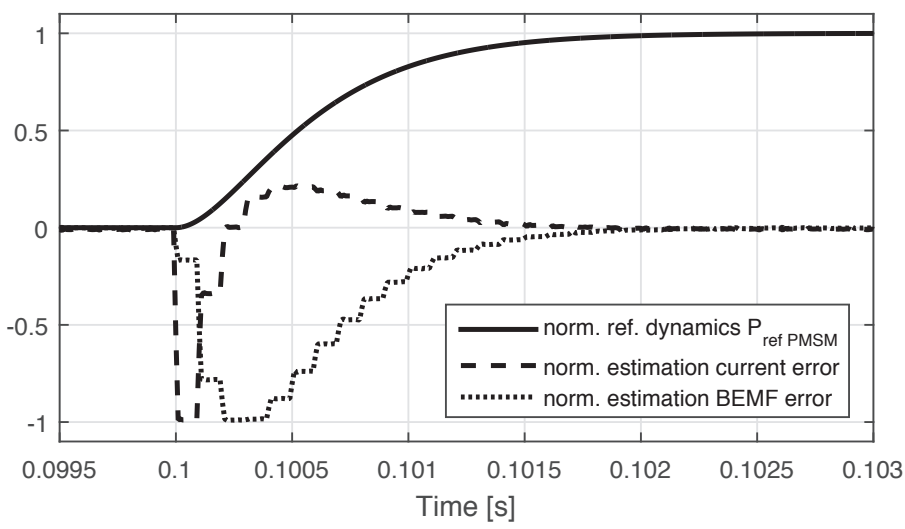

Fig. 15. Zoomed and normalized view for step response waveforms on estimation disruption, for one phase $(\alpha)$ - experiment

constantly transient state in a case of non-zero speed value. In order to obtain a clean view of decay of the estimation error, a zero speed term was chosen. This introduced disruption at time $0.1 \mathrm{~s}$ in form of step from 0 to $-10[A, V]$ results with initial error of estimated BEMF equal $-10[\mathrm{~V}]$ however, in a case of such combination of disruption, this error increases temporarily, while decreasing the current may only result in zero error. It is visible in Fig. 14, where all waveforms were normalized in order to obtain clear view. Then, estimating BEMF start to approach the real value. Despite this, estimated BEMF decays but with the designed dynamics (Fig. 14, waveform \#1).

In order to confirm propriety of presented considerations an experimental test was performed. It was used $1.23 \mathrm{~kW}$ PMSM, where $R_{s}=3 \Omega$ and $L_{s}=3.6 \mathrm{mH}$. This motor has clearly different parameters than presented in (31) but the desired dynamics of estimation error decay is the same as in (30). The experiment was carried out for reference speed $\omega_{\text {ref }}=1 \frac{\mathrm{rad}}{\mathrm{s}}$ (sensor mode). The disruption in form of step from 0 to $-10[A, V]$ was introduced. In contrast to continuous form of the observer (Fig. 11) which is used in previous examples, now a discrete form is used, with calculation period $100 \mu s$. Obtained results are shown in Fig. 15. It is clear that the referenced dynamics of estimation error decay is achieved. 


\section{Conclusions}

In the paper, a method of determining the parameters of BEMF observer was presented, in case of DC motor and PMSM. The calculation procedure of the observer parameters is directly derived from the primary materials presented by Luenberger adapted to a case of the observer for DC motor. However, to achieve the proper observer operation, this approach requires information on load torque. Its actual value must, however, be measured or estimated. In some applications it may be difficult or impossible to obtain. In such approach it is important to notice the difference in $\mathbf{C}$ matrix, in a case of model of DC motor to use in simulating (identity matrix $2 \times 2$ ), and in case of DC motor model in form to use in the observer (size $1 \times 2$ ). In addition, a whole model of object must be known, both electrical parameters and mechanical. That is why the simpler model for DC motor is prepared to build the observer. Such model does not require identifying mechanical parameters, and in particular, it does not require the information on the actual load torque. The cost of that simplification is bigger (in comparison to previous implementation) estimation errors in transients of estimated signals, in the case of the same designed dynamics. Expanding this structure to two axes, we obtain a BEMF observer which estimates BEMF in two axes independently. Such a structure becomes the observer of BEMF in $\alpha$ and $\beta$ axes for PMSM preserving the simplicity of determination the observer parameters.

Obtaining a properly working observer in open mode is a relatively simple task. It is much more difficult to obtain a properly working sensorless drive in a closed mode. Expert knowledge is needed to accomplish this stage in order to properly choose the observer dynamics and dynamics of the controllers for control chain of the drive, but that is another issue.

\section{REFERENCES}

[1] D. Luenberger, "An introduction to observers", IEEE Transactions on Automatic Control 16 (6), 596-602 (1971).

[2] A. Dębowski, D. Lewandowski, and P. Łukasiak, "Mixed-loop control of an asynchronous traction drive based on electromagnetic state stimulator concept", 2013 IEEE 18th Conference on Emerging Technologies \& Factory Automation (ETFA), 1-10 (2013).
[3] K. Urbański and K. Zawirski, "Improved method for position estimation using a two-dimensional scheduling array", Automatika 56 (3), 331-340 (2015).

[4] X. Song, J. Fang, B. Han, and S. Zheng, "Adaptive compensation method for high-speed surface PMSM sensorless drives of EMFbased position estimation error", IEEE Transactions on Power Electronics 31 (2), 1438-1449 (2016).

[5] D. Janiszewski, "Load torque estimation for sensorless PMSM drive with output filter fed by PWM converter", 39th Annual Conference of the IEEE Industrial Electronics Society (IECON 2013), 2953-2959 (2013).

[6] Z. Wang, Y. Zheng, Z. Zou, and M. Cheng, "Position sensorless control of interleaved CSI fed PMSM drive with extended Kalman filter", IEEE Transactions on Magnetics 48 (11), 36883691 (2012).

[7] K. Urbański, "Comparison of methods for back EMF estimation at low speed for PMSM drive", 2014 16th International Conference on Mechatronics - Mechatronika (ME), 32-37 (2014).

[8] F. Parasiliti, R. Petrella, and M. Tursini, "Sensorless speed control of a PM synchronous motor by sliding mode observer", Proceedings of the 1997 IEEE International Symposium on Industrial Electronics, ISIE'97, 1106-1111 (1997).

[9] P. Vaclavek and P. Blaha, "Lyapunov function based design of PMSM state observer for sensorless control", 2009 IEEE Symposium on Industrial Electronics Applications, ISIEA 2009 1, 331-336 (2009).

[10] P. Brandstetter, M. Dobrovsky, O. Petrtyl, C.S.T. Dong, and H.H. Vo, "Sensorless control of induction motor drive using BEMFMRAS with Kalman filter", 2016 ELEKTRO, 121-126 (2016).

[11] O. Benjak and D. Gerling, "Review of position estimation methods for IPMSM drives without a position sensor. Part II: Adaptive methods", 2010 XIX International Conference on Electrical Machines (ICEM), 1-6 (2010).

[12] H. Kim, J. Son, and J. Lee, "A high-speed sliding-mode observer for the sensorless speed control of a PMSM", IEEE Transactions on Industrial Electronics 58 (9), 4069-4077 (2011).

[13] J. Kabziński, "Adaptive, compensating control of wheel slip in railway vehicles", Bull. Pol. Ac.: Tech. 63 (4), 955-963 (2015).

[14] P. Vas, Sensorless Vector and Direct Torque Control, Oxford University Press, Oxford, 1998.

[15] E. Clarke, Circuit Analysis of A-C Power Systems: Symmetrical and Related Components, Wiley, New York, 1943.

[16] A. Germano, F. Parasiliti, and M. Tursini, "Sensorless speed control of a PM synchronous motor drive by Kalman filter", Proc. 1994 International Conference on Electrical Machines 2, 540-544 (1994). 